\title{
Zieve's Syndrome and Hemochromatosis, are they Related?
}

Rossi $\mathbf{S}^{*}$ and Schiavone $\mathbf{C}$

Unit of Diagnostic Ultrasound, "G. d'Annunzio" University, 66013 Chieti Scalo (CH), Italy

"Corresponding author: Rossi Stefano, Unit of diagnostic Ultrasound, "G. d'Annunzio” University, 66013 Chieti Scalo (CH), Italy, Tel: +39-871-358576; Fax: +39-871-358969; E-mail: sterota@ymail.com

Rec date: May 19, 2015 Acc date: June 10, 2015 Pub date: June 15, 2015

Copyright: (C) 2015 Rossi S, et al. This is an open-access article distributed under the terms of the Creative Commons Attribution License, which permits unrestricted use, distribution, and reproduction in any medium, provided the original author and source are credited.

\section{Case Report}

A 42 years old man with alcoholic liver cirrhosis was admitted to our hospital for the persistence of a few days of gingival bleeding, jaundice and abdominal pain. The patient reported a recent alcohol abuse. At physical examination was revealed the presence of scleral jaundice, hepatosplenomegaly, gengiva bleeding and bronzed skin. At laboratory tests was showed an increase in total bilirubin $(10.20$ $\mathrm{mg} / \mathrm{dL})$ mainly of direct fraction $(7.60 \mathrm{mg} / \mathrm{dL})$, amylase $(152 \mathrm{U} / \mathrm{L})$, lipase (936 U/L), transaminases (GOT $400 \mathrm{U} / \mathrm{L}$ and GPT $165 \mathrm{U} / \mathrm{L}$ ), GGT (326 U/L), alkaline phosphatase (333 U/L), PCR $6.2 \mathrm{mg} / \mathrm{dL}$, macrocytic anaemia (Hb $7.7 \mathrm{~g} / \mathrm{dL}, \mathrm{MCV} 109.5 \mathrm{fL}$ ), INR 2.

Moreover, further exams were performed:

- Study of iron status that showed a significant increase in serum ferritin $(4144 \mathrm{mcg} / \mathrm{L}$, so it was performed the genetic study that affirmed the alcoholic etiology of the hemochromatosis)

- Peripheral smear showed the presence of schistocytosis

- Haptoglobin assay (undetectable)

- Direct Coombs test (negative) and indirect Coombs (positive)

- Reticulocytes with physiological increase after anaemia

- TORCH, HBV and HCV negative

- ANA, ENA, anti-LKM, anti-SMA anti SLA, anti-AMA negative

Sonography with ecocolor excluded the presence of biliary obstruction but showed signs of chronic cirrhosis complicated from portal hypertension.

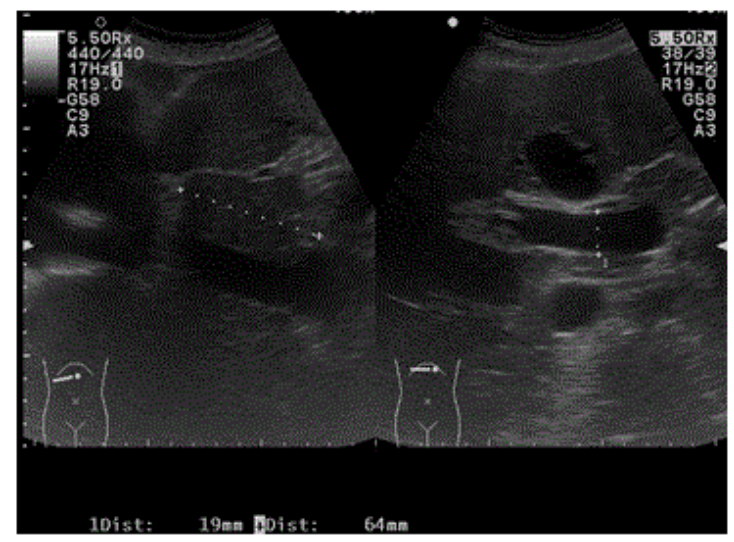

Figure 1: First lobe and portal vein size

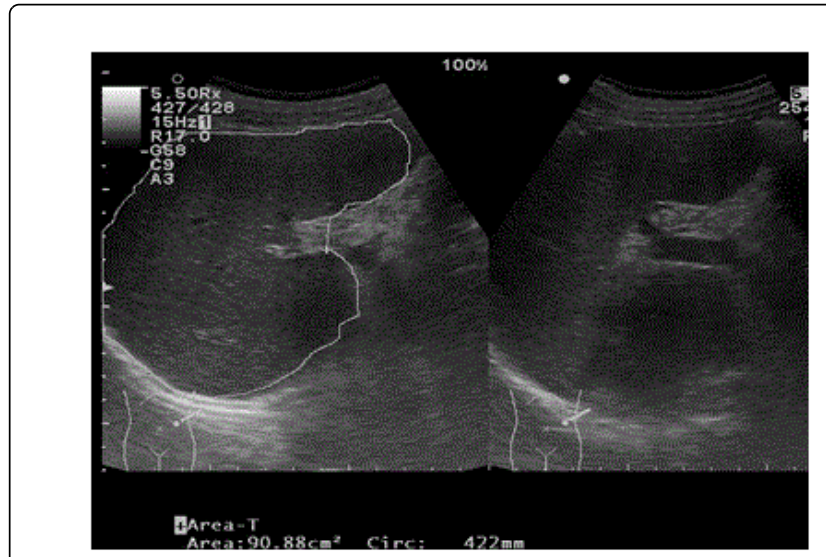

Figure 2: Splenic area.
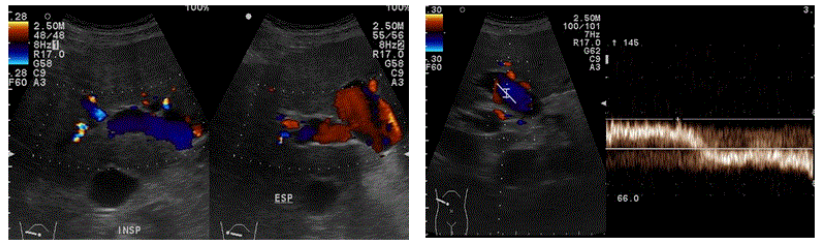

Figure 3: Portal biphasic flow with breaths at colour and spectral analysis associated with umbilical vein recanalization.

At the EGDS was showed oesophageal varices (grade I) in the context of a congestive gastropathy and antral gastritis. So the patient was treated with fasting, abstaining from alcohol and fluids therapy, branched amino acids and gastric protection with progressive improvement in clinical exams and laboratory tests. Furthermore, it was referred to a center for alcohol detoxification and start iron chelation therapy. At the end the patient was discharged with a diagnosis of alcoholic cirrhosis in Zieve syndrome and secondary hemochromatosis from alcohol abuse (Figures 1-3).

\section{Discussion}

Zieve's syndrome, described for the first time by Leslie Zieve in 1958 , it is characterized by the presence of jaundice, haemolytic anaemia, increased transaminases and transient hyperlipidemia associated with alcohol abuse [1]. Zieve saw that after interruption of alcohol consuming, jaundice and hypercholesterolemia improved 
Page 2 of 3

quickly. There aren't pathognomonic symptoms or clinical signs of the syndrome, so it is difficult to recognize this disease. A further cause for underdiagnosing of this illness may be that hyperlipidemic status rapidly decreases after stop alcohol intaken.

Some authors showed that hyperlipidemia stopped before the appearance of haemolysis that is caused by an acquired deficiency of pyruvate kinase blood red cells's. This finding is absent in patient with alcoholic chirrosis uncomplicated from haemolysis [2,3]. We have shown a case of Zieve syndrome without hyperlipidemia because the patient was being treated with simvastatina $40 \mathrm{mg}$. This therapy was set few years before in another hospital admission where he showed, after an alcohol abuse, the same clinical and laboratory findings but this time associated with hyperlipemia (total cholesterol $210 \mathrm{mg} / \mathrm{dL}$ ).

Zieve's syndrome can occur, also, in patients with or without preexistent alcohol liver damage. The diagnosis requires exclusion of viral, autoimmune hepatitis and biliary obstruction; negativity of serologic and autoimmune markers. Hemochromatosis is an abnormal accumulation of iron in parenchymal organs of the body that leads, if not treated to organ dysfunction $[4,5]$. Iron overload syndromes are divided into two main groups (Tables 1 and 2).

\begin{tabular}{|l|l|}
\hline $\begin{array}{l}\text { HFE related hemochromatosis } \\
\text { (Type 1) }\end{array}$ & Primary \\
\hline & C282Y/C282Y \\
\hline & C282Y/H63D \\
\hline & $\begin{array}{l}\text { Other HFE mutations Non-HFE related } \\
\text { hemochromatosis } \\
\text { Hemochromatosis (Type 2) }\end{array}$ \\
\hline & Type 2A-Hemojuvelin mutations \\
\hline $\begin{array}{l}\text { Transferrin receptor } \\
\text { hemochromatosis (Type 3) } \\
\text { Ferroportin diseases (Type 4) }\end{array}$ & Type 2B - Hepcidin mutations \\
\hline & Classical \\
\hline & Nonclassical \\
\hline
\end{tabular}

Table 1: Iron overload syndromes - Primary.

\begin{tabular}{|c|c|}
\hline & Secondary \\
\hline \multicolumn{2}{|l|}{$\begin{array}{l}\text { Iron-loading } \\
\text { anemias }\end{array}$} \\
\hline & Thalassemic syndromes (b Thalassaemia) \\
\hline & Sideroblastic Anaemias \\
\hline & Chronic Haemolytic Anaemia \\
\hline & Aplastic Anaemia \\
\hline & Pyruvate Kinase Deficiency \\
\hline \multicolumn{2}{|l|}{$\begin{array}{ll}\text { Chronic } & \text { liver } \\
\text { disease } & \end{array}$} \\
\hline & Hepatitis C infection \\
\hline & Porphyria Cutanea Tarda \\
\hline
\end{tabular}

\begin{tabular}{|l|l|}
\hline & Alcoholic liver disease \\
\hline & NAFLD latrogenic \\
\hline & Red Blood cell transfusion \\
\hline Miscellaneous & Long-term haemodialysis \\
\hline & Aceruloplasminaemia \\
\hline & African iron overload \\
\hline & Neonatal iron overload \\
\hline
\end{tabular}

Table 2: Iron overload syndromes - Secondary.

Hemochromatosis is still underdiagnosed by clinicians because it is considered a rare disorder. The majority of patients with hemochromatosis appear asymptomatically but when they become symptomatic may present with: fatigue, right upper quadrant abdominal pain, arthralgias, (typically of the second and third metacarpophalangeal joints), chondrocalcinosis, impotence, decreased libido, and symptoms of heart failure or diabetes. Physical findings depends of organ damage: an enlarged liver, signs of cirrhosis, testicular atrophy, diabetes, congestive heart failure, skin pigmentation, changes of porphyria cutanea tarda, and arthritis [6].

At physical examination, our patient showed with bronzed skin and clinical signs of liver cirrhosis (hepatosplenomegaly, eversion of the umbilicus, ascites, thrombocytopenia), which added to the results of laboratory tests, induced us to suspect the diagnosis of alcoholic hemochromatosis, later confirmed by genetic testing. In fact, the diagnosis of hemochromatosis is based on evidence of increased iron stores, demonstrated by elevated serum ferritin levels, which reflects an increase in hepatic iron content.

The hereditary hemochromatosis can be defined genotypically by the familial occurrence of iron overload associated with some genetic mutation (C282Y homozygosity or C282Y/H63D compound heterozygosity). Liver biopsy should be considered only for demonstrate the presence or absence of advanced fibrosis or cirrhosis, which does have prognostic value.

The treatment of hemochromatosis is based on the removal of iron excess that can be made with phlebotomy or with the use of chelating drugs.

\section{Conclusions}

Zieve's syndrome is a rare condition characterized by haemolytic anaemia, jaundice and hyperlipidemia after alcohol abuse in patients with chronic liver disease [3]. So, in these patients, it must be considered in the differential diagnosis of certain unknown liver injury, haemolysis or hemorragias.

The relation between liver iron stores and alcoholic liver disease is well demonstrated since the 1964 , and hemochromatosis is believed to be a particular manifestation of alcoholic cirrhosis $[7,8]$. The liver iron surplus is an independent risk factor for fibrosis, while in alcoholic cirrhosis the accumulated liver iron concentrations correlate inversely with patient survival. It is known that serum iron and ferritin increase linearly with daily alcohol consumption [9]. 
Citation: Rossi S, Schiavone C (2015) Zieve's Syndrome and Hemochromatosis, are they Related?. General Med 3: 181. doi:

In our case we have showed that alcohol is the string that joins two rare illnesses, because: after an acute alcohol abuse our patient have manifested a Zieve's syndrome, but is exactly the chronic alcohol consumption that has caused a secondary hemochromatosis and cirrhosis. So we can concluded that even in the face of a common disease as alcoholic cirrhosis we should ask ourselves if it hides a rare diseases, because the treatment requires not only the stop of alcohol consumption but also additional measures, such as the use of chelating agents to avoid further organ damage.

\section{References}

1. Zieve L (1958) Jaundice, hyperlipemia and hemolytic anemia: a heretofore unrecognized syndrome associated with alcoholic fatty liver and cirrhosis. Ann Intern Med 48: 471-496.

2. Melrose WD, Bell PA, Jupe DM, Baikie MJ (1990) Alcohol-associated haemolysis in Zieve's syndrome: a clinical and laboratory study of five cases. Clin Lab Haematol 12:159-167.
3. Jūratė K, Inga B, Sigita G, Limas K (2012) Zieve's syndrome presenting with coagulopathy, skin and subcutaneous haemorrhage Acta Medica Lituanica 19: 403-407.

4. Fleming RE, Sly WS (2002) Mechanisms of iron accumulation in hereditary hemochromatosis. Annu Rev Physiol 64:663-680.

5. Siddique, Kowdley KV (2012) Review article: the iron overload syndromes. Aliment Pharmacol Ther 35:876-893.

6. Bruce R Bacon, Paul C Adams, Kris V Kowdley, Lawrie W Powell, Anthony S (2011) Diagnosis and Management of Hemochromatosis: 2011 Practice Guideline by the American Association for the Study of Liver Diseases. Hepatology 54: 328-343.

7. Fletcher LM, Bridle KR, Crawford DH (2003) Effect of alcohol on iron storage diseases of the liver. Best Pract Res Clin Gastroenterol. 17:663-677.

8. Fletcher LM, Powell LW (2003) Hemochromatosis and alcoholic liver disease. Alcohol 30:131-136.

9. Whitfield JB, Zhu G, Heath AC, Powell LW, Martin NG (2001) Effects of alcohol consumption on indices of iron stores and of iron stores on alcohol intake markers. Alcohol Clin Exp Res 25:1037-1045. 